\title{
Perceptions des enseignants et des garçons à l'égard de la relation enseignant-élève au secondaire : quand les stéréotypes de genre s'immiscent en classe
}

\author{
Carl Beaudoin \\ Université de Sherbrooke
}

\section{Résumé}

Cet article ${ }^{1}$ vise à identifier les stéréotypes de genre véhiculés par les enseignants de sexe masculin et les garçons du secondaire dans leurs perceptions déclarées à l'égard de leurs relations enseignants-élèves (REÉ). Se situant dans une approche qualitative interprétative, cette étude met en évidence les propos de 18 enseignants et de 86 garçons du secondaire, collectés par le biais de trois stratégies de collecte de données, soit l'entrevue semi-dirigée, le groupe de discussion et le questionnaire d'enquête. Les données qualitatives obtenues ont été analysées à l'aide des catégories conceptualisantes. Les principaux résultats mettent en lumière que certains enseignants mentionnent communiquer ou intervenir auprès des

1 Cet article est issu d'un projet de recherche financé par le Fonds de recherche du Québec - Société et culture (FRQSC).

Canadian Journal of Education / Revue canadienne de l'éducation 44:3 (2021)

(c)2021 Canadian Society for the Study of Education/ Société canadienne pour l'étude de l'éducation 
garçons sur la base d'intérêts stéréotypés masculins. Quant aux garçons, plusieurs d'entre eux font ressortir la distanciation émotive des enseignants à leur égard.

Mots-clés : stéréotypes de genre, relation enseignant-élève, intérêts stéréotypés, masculinité traditionnelle, intervention genrée

\begin{abstract}
This article aims to identify the gender stereotypes conveyed by male teachers and male high school students in their stated perceptions of their teacher-student relationships (TSR). Using an interpretative qualitative approach, this study highlights the statements of 18 teachers and 86 boys in high school collected through three data collection strategies, namely the semi-structured interview, the focus group and the survey questionnaire. The qualitative data obtained were analyzed using the conceptualizing categories. The main results highlight that some teachers mention communication with boys is based on stereotypical male interests. In counterpart, some boys report the teachers being emotionally distant from them.
\end{abstract}

Keywords: gender stereotypes, teacher-student relationship, stereotypical interests, traditional masculinity, gendered intervention 


\section{Introduction et problématique}

Depuis quelques années, les instances gouvernementales, les médias et certains chercheurs au Québec et dans plusieurs pays occidentaux soulèvent l'hypothèse d'un lien possible entre la faible représentativité des hommes en enseignement et certaines difficultés sociales ou scolaires des garçons (Carrington et al., 2008 ; Francis, 2008 ; Royer, 2010, 2011). En consultant les données pour le Québec, on ne peut que remarquer la faible présence des hommes en éducation. En effet, en enseignement secondaire, environ $37 \%$ des membres du personnel enseignant étaient des hommes en 20152016 (Ministère de l'Éducation et de l'Enseignement supérieur [MEES], 2018). Pour la même période, en enseignement primaire, on observe que les hommes représentent seulement autour de $12 \%$ du personnel enseignant (MEES, 2018). Alors que s'entament ces réflexions entourant le manque de modèles masculins chez le personnel enseignant en milieu scolaire, des décideurs politiques, des chercheurs et des acteurs du milieu scolaire se sont mobilisés afin de favoriser la persévérance scolaire chez les élèves, et particulièrement chez les garçons qui sont moins nombreux à obtenir un diplôme que les filles. En effet, le taux de diplomation dans le réseau public demeure relativement faible chez les garçons : seulement $43 \%$ d'entre eux réussissent le programme de deuxième cycle du secondaire en 5 ans, comparativement à $57 \%$ pour les filles (Homsy et Savard, 2018). D’ailleurs, « l'écart s'accroît entre les sexes dans les milieux défavorisés sur le plan socioéconomique parce que les garçons s'associent plus fortement aux stéréotypes masculins dans lesquels le goût et le désir de se scolariser sont souvent absents » (Lamarre, 2019, p. 130).

À notre connaissance, les recherches publiées actuellement ne montrent pas la présence d'une relation directe entre le sexe de l'enseignant et la réussite scolaire chez les garçons (Carrington et al., 2007 ; Hansen et Quintero, 2018). D’ailleurs, les élèves, garçons ou filles, disent avant tout s'intéresser aux aptitudes en gestion de classe du personnel enseignant (Carrington et al., 2007). De l'avis de certains auteurs, les enseignants de sexe masculin sont identifiés comme des modèles positifs pour les garçons dans leur construction identitaire et comme des confidents dans le rapport des garçons avec la puberté (McGrath et Sinclair, 2013). Ainsi, il y a lieu de penser que les garçons qui s'identifient et se confient à leurs enseignants ont développé une relation enseignantélève (REÉ) significative qui leur permet de se sentir en confiance. 
À ce sujet, les chercheurs ont examiné les questions relatives à la REÉ pour mieux décrire la situation des garçons à l'école. De nombreuses études ont traité de la REÉ sur le plan affectif en s'appuyant principalement sur la théorie de l'attachement de Bowlby (1969), qui montre que la relation d'attachement que l'enfant développe avec sa mère ou un adulte significatif présente des similitudes avec celle qu'il établira ultérieurement avec le personnel enseignant. Numa-Bocage (2007) s'est quant à elle intéressée à la description de la REÉ sur le plan cognitif, soutenant que les apprentissages qu'effectue l'enfant à l'école se développent en partie à travers les échanges avec le personnel enseignant. Notons toutefois que plusieurs auteurs se sont déjà penchés sur l'aspect cognitif de l'apprentissage en faisant des liens plus ou moins explicites avec le concept de la REÉ en soulignant, entre autres, que le développement cognitif et langagier de l'enfant est tributaire des dispositions prises par les adultes pour faciliter l'expression des compétences du jeune et l'encourager dans le développement de compétences encore plus complexes (Davis et Miyake, 2004 ; Good et Brophy, 2017 ; Skibbe et al., 2004). Partant de la prémisse que tout comportement opératoire qu'on désire apprendre ou faire apprendre est socialement situé (Postic, 2001), il s'avère pertinent de considérer également la REÉ sur le plan social, puisque ce dernier sert en quelque sorte de réceptacle à ses aspects affectif et cognitif. En fait, on peut difficilement faire fi du contexte social dans lequel la REÉ se développe et est traversée, notamment par l'héritage culturel, la mise en œuvre de méthodes de travail, de pensées et d'actions ancrées dans les mœurs et le contexte politique (Durkheim, 2014).

\section{Cadre de référence}

\section{La relation enseignant-élève comme phénomène social}

Tout système scolaire porte la marque de la société qui l'a produit : il est organisé selon la conception de la vie sociale, des rouages de la vie économique et des rapports sociaux qui animent cette société (Durkheim, 2014 ; Rayou, 2017). Ce sont d'ailleurs dans les rapports sociaux présents dans l'acte éducatif que le personnel enseignant et les élèves se découvrent et évoluent. Il en découle des jeux de force ou de pouvoir où la REÉ se resserre, se situe ou se distend. Le conflit y est présent et des crises sont susceptibles de 
surgir, parfois contenues, parfois occultées (Durkheim, 2014). Autrement dit, l'éducation se calquerait sur la division des classes sociales et des luttes de pouvoir, ce qui influencerait la dynamique même de la REÉ. Sous le couvert de la pensée durkheimienne, la REÉ est conçue de manière asymétrique : l'élève a un rôle passif puisqu'il n'a pas suffisamment de représentations, c'est-à-dire de connaissances et d'expérience pour rivaliser avec celles du personnel enseignant (Durkheim, 2014 ; Rayou, 2017). Il se voit contraint d'accepter et de croire uniquement l'enseignement qui lui est prodigué. Quant au personnel enseignant, c'est la supériorité de l'expérience de sa culture qui le place dans un rôle actif. Détenant le savoir et connaissant les rouages du système d'éducation, le personnel enseignant détient une forme de pouvoir qu'il exerce dans ses relations avec l'élève.

Bien que dans cette représentation de la REÉ, la passivité de l'élève semble immuable, Durkheim (2014) souligne qu'elle ne l'est pas : les apprentissages que fera l'élève de même que son expérience qui se développera au fil du temps lui permettront de défier l'expérience du personnel enseignant. Cette défiance est en quelque sorte une tentative pour fragiliser ou revendiquer le pouvoir. Elle peut s'exprimer par le biais de comportements nuisibles des élèves, qui chahuteront le personnel enseignant ou encore se mureront dans le silence lorsqu'on leur demandera de participer (Pujade-Renaud, 2005). D'ailleurs, les élèves peuvent s'allier contre le personnel enseignant en générant des attitudes hostiles à son égard afin de démontrer leur force comme groupe social (Passerieux, 2010 ; Willis, 2011).

Selon Postic (2001) et Rayou (1998), le personnel enseignant demeure un agent de reproduction sociale censé préserver l'ordre établi. Cet ordre est gardé grâce aux connaissances détenues et transmises par le personnel aux élèves ainsi qu'au maintien et à la reproduction des rapports hiérarchisés qui s'établissent à l'école (Rayou, 1998). Ainsi, plusieurs aspects sociaux viennent influencer la REÉ : il suffit de penser aux règles établies à l'école, au programme scolaire qui y est prévu de même qu'à l'environnement social dans lequel se déroulent les relations (Forquin, 2008 ; Montandon, 2002). En fait, l'école, à travers les activités qu'elle préconise, s'inscrit dans un système d'attitudes, de manières de penser, d'intérêts et de valeurs qui ne sont pas socialement neutres (Forquin, 2008 ; Rayou, 2017 ; Rey, 1999).

Outre l'école, le travail scolaire effectué par les élèves témoigne également des forces sociales qui s'exercent dans la REÉ. Au dire de Clément et al. (2011) et de 
Qvortrup (1995), les élèves sont entrainés à être utiles pour la société, à constituer une force productrice susceptible de rejoindre le marché du travail. Baby (2016) abonde en ce sens en mentionnant que l'école québécoise n'échapperait pas aux influences néolibérales qui visent à rendre avant tout les élèves aptes à intégrer le marché du travail et qu'elle devrait plutôt les former à devenir de futurs citoyens pensants et critiques de la société dans laquelle ils évoluent. De leur côté, Tardif et Gauthier (2017) ont une vision humaniste de l'école québécoise. Ils sont d'ailleurs d'avis que la REÉ s'est transformée au cours du 20e siècle, entre autres influencée par la reconnaissance du droit des enfants (p. ex., droit d'apprendre, d'être respectés par les adultes). Aujourd'hui, l'idée d'obéissance et de passivité à l'égard du maître est presque révolue dans la REÉ au Québec (Tardif et Gauthier, 2017).

Si le contexte social s'avère important pour opérationnaliser la REÉ, il appert que le phénomène des stéréotypes de genre est sujet à influencer cette dynamique des rapports sociaux dont il a été question entre le personnel enseignant et les garçons et, par ricochet, à se traduire dans leurs perceptions à l'égard de la REÉ.

\section{Les stéréotypes de genre en classe}

Les stéréotypes de genre sont des mécanismes psychologiques qui, sans que nous en ayons conscience, orientent et altèrent notre regard, notre jugement sur autrui, nos interprétations ainsi que nos attentes à l'égard des hommes et des femmes, venant en quelque sorte justifier la division sociale sur la base du sexe des individus (Mosconi, 2017 ; Prentice et Carranza, 2002). Selon Bréau et Lentillon-Kaestner (2016), les

attitudes ou les comportements socialement attendus pour les hommes et pour les femmes contiennent bon nombre de prescriptions ou d'interdits, souvent implicites, auxquels ils et elles tenteront d'adhérer dans le but de préserver leur référent identitaire et leur sentiment d'appartenance au groupe. L'existence des stéréotypes de genre constituerait un obstacle au développement plein et entier des aptitudes des hommes et des femmes (Duru-Bellat, 2016). En Occident, le masculin renvoie généralement à la force physique, tandis que le féminin est souvent associé à l'émotivité, ce qui illustre un rapport déséquilibré entre les deux groupes de sexe, où le masculin est signe de force et le féminin, de faiblesse (Castillo-Mayén et Montes-Berges, 2014). À ce sujet, lorsqu'une collectivité s'attend à ce que les hommes se montrent indépendants, suffisants et cachent leurs sentiments, 
cela peut engendrer une atrophie affective ou émotionnelle, un manque d'ouverture, une incapacité à reconnaître la vulnérabilité et une déficience sur le plan de la communication interpersonnelle pour les hommes (Blais et al., 2019). En effet, Anderson (2009) et Connell (1995) mentionnent que l'archétype masculin traditionnel dans les sociétés occidentales contemporaines qualifie de masculinité orthodoxe l'inhibition émotionnelle attendue des hommes. Elle se manifeste notamment par la distanciation émotionnelle à l'égard des femmes et des hommes, et par la distanciation physique à l'égard des autres hommes. Quant aux femmes, lorsque la société s'attend à ce qu'elles soient émotives, sensibles, attentionnées, dépendantes et non violentes, comme c'est le cas dans la plupart des cultures occidentales, elle les maintiendrait dans des rôles de subalternes (Lips, 2005).

Lorsqu'il est question du contexte scolaire, quelques auteurs mentionnent que le personnel enseignant peut être influencé par ses valeurs personnelles et ses préjugés culturels (Davis et Rimm, 2004 ; Erbas et al., 2010) et opter pour des comportements stéréotypés auprès des élèves de couleur (Harrison, 2011 ; Riley et Ungerleider, 2012 ; Van Ewijk, 2011), en difficulté d'apprentissage (Bianco et Leech, 2010) ou issus d'un milieu socioéconomique défavorisé (McBee, 2006). Des études révèlent que le personnel enseignant perçoit généralement les filles comme étant plus susceptibles de faire des efforts et de s'engager en classe comparativement aux garçons (Burusic et al., 2012 ; Clark et al., 2008). De plus, des membres du personnel enseignant considéreraient les garçons comme étant davantage désengagés sur le plan scolaire (Auwärter et Aruguete, 2008 ; Legewie et DiPrete, 2012) et plus portés à manifester des comportements inappropriés en classe comparativement aux filles (Clark et al., 2008). D'autres études montrent que le personnel enseignant continue d'entretenir des stéréotypes de genre qui risquent d'avoir des conséquences négatives sur les élèves (Duru-Bellat, 2016), notamment lorsque les garçons sont identifiés comme étant plus actifs et agressifs que les filles, qui elles, sont reconnues comme étant d'emblée plus coopératives et agréables (Mosconi, 2017). Enfin, le personnel enseignant attribue la réussite scolaire des filles à leurs efforts, alors que pour les garçons, c'est plutôt à leur « habileté naturelle » (Quenzel et Hurrelmann, 2013). 


\section{L'objectif poursuivi}

Dans le contexte où l'incidence des stéréotypes de genre est susceptible de caractériser spécifiquement la REÉ, plus précisément entre les enseignants et les garçons, cet article a pour objectif d'identifier les stéréotypes de genre véhiculés par les enseignants et les garçons dans leurs perceptions déclarées à l'égard de la REÉ.

\section{Méthode}

S'inscrivant dans une approche qualitative interprétative, cette étude vise à accéder plus aisément à la compréhension du sens que donnent les enseignants et les garçons à leurs expériences relationnelles. Nous avons ainsi cherché à dégager de ces expériences des stéréotypes de genre à partir des perceptions déclarées des enseignants et des garçons à l'égard des relations qu'ils entretiennent mutuellement. Dans ce qui suit, nous traiterons de l'échantillon à l'étude de même que des stratégies de collecte et d'analyse des données.

\section{Les participants}

Afin de recruter nos participants, soit des enseignants et des élèves de sexe masculin, nous avons privilégié les classes d'enseignement régulier et d'adaptation scolaire et sociale du secondaire de la région du Centre-du-Québec. Après avoir sollicité par téléphone ou par courriel les directeurs d'école de la région ciblée, deux d'entre eux ont accepté de prendre part à la recherche et de la soumettre aux membres de leur personnel enseignant. Au total, notre échantillon de convenance est constitué de 18 enseignants âgés de 28 à 50 ans (Médiane $=35$ ans), œuvrant en 2e secondaire, toutes matières confondues, ainsi que de leurs élèves, soit 86 garçons âgés de 12 à 13 ans.

\section{Les stratégies de collecte de données}

A priori, nous avions envisagé de recourir à une seule stratégie de collecte de données, soit les entrevues semi-dirigées. Il nous a cependant fallu nous adapter aux demandes des répondants qui préféraient participer, mais par l'entremise d'un groupe de discussion ou encore d'un questionnaire d'enquête. Sans ces changements, les participants se 
seraient retirés de l'étude. D'ailleurs, cette prise en compte des besoins des répondants sur le plan de la collecte des données est conforme à la logique de l'approche qualitative interprétative empruntée par la présente étude (Karsenti et Savoie-Zajc, 2018). Comme le montre le tableau 1, les enseignants et les garçons n'ont participé qu'à l'une ou l'autre des stratégies de collecte de données. Par souci de transparence, mentionnons que les questions relatives à la REÉ et aux stéréotypes de genre, que ce soit pour le schéma d'entrevue semi-dirigée et de groupe de discussion ou pour le questionnaire d'enquête, ont été en partie inspirées des dimensions du modèle de gestion de classe de Pianta et al. (2012), soit le climat de classe, la sensibilité de l'enseignant, la gestion des comportements, la compréhension du contenu enseigné et la qualité des rétroactions.

\section{Tableau 1}

Répartition des participants selon la stratégie de collecte de données

\begin{tabular}{lcc}
\hline Stratégie de collecte de données & Enseignants & Garçons \\
\hline Entrevue semi-dirigée & 5 & 8 \\
Groupe de discussion & 10 & 24 \\
Questionnaire d'enquête & 3 & 54 \\
\hline Total & 18 & 86 \\
\hline
\end{tabular}

L'entrevue semi-dirigée. Nous avons eu recours à l'entrevue semi-dirigée, principalement parce que nous voulions « être en mesure de dépasser les thèmes ou les questions déjà formulés au schéma d'entrevue et de vérifier auprès [des enseignants et des garçons], si la compréhension émergente des expériences partagées [était] adéquate » (Savoie-Zajc, 2010, p. 355). Nous avons d'ailleurs abordé les thèmes ou les questions « selon l'ordre et la logique des propos tenus pendant la rencontre [...] tout en assurant une certaine constance d'une entrevue à l'autre, même si l'ordre et la nature des questions, les détails abordés et sa dynamique particulière peuvent [avoir différé] » (Savoie-Zajc, 2018, p. 200). Ainsi, parmi le personnel enseignant, 5 hommes ont été rencontrés individuellement et près de 20 questions ouvertes leur ont été posées. Quant aux élèves, 8 garçons ont participé individuellement aux échanges lors desquels nous leur avons posé 12 questions ouvertes. 
Le groupe de discussion. Nous avons également privilégié le groupe de discussion. Concernant les enseignants, le schéma de groupe de discussion comportait près de 20 questions ouvertes que nous avons soumises à 2 groupes, pour un total de 10 hommes. Quant aux élèves, nous avons rencontré au total 24 garçons. Nous avons ainsi formé six groupes de discussion composés de quatre garçons chacun. Une dizaine de questions ouvertes leur ont été soumises. Ce type d'exercice nous a permis d'approfondir les réponses des participants en les invitant à les expliquer plus en détail, à « relever les expériences vécues qui ont contribué à former [leurs] opinions, élucider les émotions et les sentiments sous-jacents à certains énoncés »(Geoffrion, 2009, p. 392).

Le questionnaire d'enquête. En complémentarité avec l'entrevue semi-dirigée et le groupe de discussion, cette stratégie s'est avérée pertinente puisque certains participants ont signifié leur « besoin de réflexion, de temps ou d'intimité pour répondre [aux questions qui leur étaient posées] » (Blais et Durand, 2009, p. 467-468). Du côté des enseignants, le questionnaire d'enquête, comprenant une quinzaine de questions ouvertes, a été rempli par trois hommes. Ces derniers avaient la possibilité d'effectuer cette tâche à la maison ou à l'école. Nous aurions souhaité recueillir davantage de données à l'aide de questionnaires auprès des enseignants, mais plusieurs d'entre eux nous ont été retournés vierges en raison d'un manque de temps de leur part. Quant aux élèves, 54 garçons ont répondu au questionnaire d'enquête constitué d'une dizaine de questions ouvertes. Une période de 30 minutes leur a été allouée pour le remplir en classe.

\section{L'analyse des données}

L'ensemble des données recueillies auprès des enseignants et des garçons a formé un corpus de 1500 pages qui ont fait l'objet d'une analyse qualitative réalisée au moyen du logiciel NVivo. Suivant la tradition de la théorisation ancrée (Charmaz, 2006 ; Glaser et Strauss, 1967 ; Strauss et Corbin, 2004), l'analyse à l'aide des catégories conceptualisantes (Paillé et Mucchielli, 2008) a été privilégiée dans le cadre de cette recherche. Elle consiste en une description du phénomène, qui passe par une hiérarchisation conceptuelle (Paillé et Mucchielli, 2016 ; Strauss et Corbin, 2004). Ce phénomène, dans le cas qui nous préoccupe, prend place dans les stéréotypes de genre véhiculés par le personnel enseignant et les garçons à l'égard de la REÉ, et 
requiert donc une stratégie d'analyse des données susceptible de bien les cerner et de les opérationnaliser, ce que, par exemple, une analyse thématique n'aurait pas permis de faire, puisque les stéréotypes de genre s'immiscent dans le discours et ne sont pas mentionnés explicitement par les participants. Afin de procéder à la formation de catégories en regard du corpus, nous avons identifié les phénomènes présents en nous questionnant, comme nous invitent à le faire Paillé et Mucchielli (2008) : «Compte tenu de ma problématique, quel est ce problème ? [...] Comment puis-je le nommer conceptuellement? »(p. 234). Nous avons donc procédé à l'examen des catégories sur l'ensemble de notre corpus en nous engageant dans une tentative de recherche de sens émanant des propos formulés par le personnel enseignant et les garçons. La catégorie est l'expression en mots de la forme de l'expérience et non du témoignage des participants : elle est porteuse du sens qui lui est conféré (Paillé et Mucchielli, 2016). Afin d'accompagner le lecteur dans la compréhension de la démarche de formulation d'une catégorie, voyons ici quelques exemples possibles : elle révèle un vécu (sentiment d'abandon), un état (paralysie de la négociation relationnelle), une action (appropriation collective de l'espace), un processus (désinstitutionnalisation), un incident situationnel (rupture de la routine pédagogique), une logique et une dynamique (contre-dépendance affective) (Paillé et Mucchielli, 2016). En plus de procéder à la formulation de catégories, nous avons défini leur sens donné. Par la suite, nous avons effectué la spécification des propriétés de la catégorie, ce qui a consisté en l'extraction des éléments qui caractérisent le phénomène des stéréotypes de genre présent dans la catégorie. En dernier lieu, un exercice de description des conditions d'existence de la catégorie a été mené afin de circonscrire les raisons de la matérialisation du phénomène des stéréotypes de genre dans son contexte (Paillé et Mucchielli, 2016).

\section{Résultats}

Dans ce qui suit, nous présentons les résultats issus de l'analyse par catégories conceptualisantes visant à identifier les stéréotypes de genre véhiculés par les enseignants et les garçons dans leurs perceptions déclarées à l'égard des relations qu'ils entretiennent mutuellement. Soulignons que des extraits de corpus ont été insérés afin d'appuyer les résultats. Dans le but de repérer ces extraits et de les associer au bon participant, nous 
les avons identifiés à l'aide d'un code alphanumérique. Par exemple, la mention PE1-2010 désigne le numéro attribué au personnel enseignant pour lequel nous avons choisi un extrait se situant à la ligne 20 de la page 10 du corpus. Il en sera de même lorsqu'il sera question des garçons, où la mention PE sera remplacée par G.

\section{Les stéréotypes de genre véhiculés par les enseignants}

Les stéréotypes de genre véhiculés par les enseignants dans leurs perceptions déclarées se traduisent par trois catégories conceptualisantes, soit la conscientisation en devenir, la communication genrée de même que l'interventionnisme genré.

La conscientisation en devenir. La conscientisation en devenir se définit comme une prise de conscience inachevée et non intériorisée de la part des enseignants quant à la présence de stéréotypes de genre en classe.

\section{Tableau 2}

Conscientisation en devenir

Catégorie : Conscientisation en devenir

Définition : Prise de conscience inachevée et non intériorisée de la présence de stéréotypes de genre en classe

\section{Propriétés :}

- Faible mobilisation dans l'enseignement concernant les stéréotypes

- Analyse réflexive à l'égard de l'intégration ou non de stéréotypes dans les pratiques enseignantes actuelles

- Poursuite de pratiques orientées en fonction d'attentes genrées de la part des élèves

\section{Conditions d'existence :}

- Craintes relatives au changement

- Nécessité d'en apprendre encore davantage sur l'incidence des stéréotypes de genre

- Besoin de temps pour envisager les changements possibles dans les pratiques enseignantes

Comme l'indique le tableau 2, des enseignants mobilisent peu d'actions ou d'efforts en classe pour faire état de la situation, à savoir s'ils sont porteurs de stéréotypes ou encore s'ils interviennent à cet égard en classe. En d'autres mots, ils poursuivent leurs réflexions sur le sujet et perpétuent implicitement des pratiques orientées en fonction d'attentes genrées auprès des garçons : « Mais là, ça fonctionne bien comment je gère 
ou j'enseigne dans ma classe... donc, je ne sais pas si ça vaudrait vraiment la peine que j'essaie de quoi de nouveau » (PE13-22-341).

Bien honnêtement, je ne me suis jamais vraiment arrêté à ça... j’agis en fonction de ce que je ressens ou je vois en classe... j'ai sans doute des attitudes particulières avec les gars et avec les filles... il faut se rappeler... je suis un gars... donc c'est normal que j'agisse différemment avec les filles... qu'avec les gars. Pas évident comme question, hein ? [rires]. (PE11-19-242)

Cette conscientisation reste en devenir parce que des enseignants expriment des craintes relatives au changement. Pour le moment, ils demeurent persuadés que le statu quo sur cette question est envisageable, puisqu'ils ne perçoivent pas les incidences que peuvent avoir les stéréotypes de genre dans la gestion ou les pratiques exercées en classe. Certains enseignants témoignent tout de même de leur ouverture et s'engagent à en apprendre davantage sur le sujet. Ils indiquent que du temps leur est nécessaire pour envisager des changements éventuels dans leurs pratiques.

La communication genrée. La communication genrée se rapporte aux échanges verbaux ou non verbaux stéréotypés masculins entre les enseignants et les garçons.

\section{Tableau 3}

\section{Communication genrée}

Catégorie : Communication genrée

Définition : Échanges verbaux ou non verbaux stéréotypés masculins

Propriétés :

- Discussions à l'accueil orientées autour des loisirs et des sports

- Attitude dynamique auprès des garçons

- Salutations sous la forme de poignées de main

\section{Conditions d'existence :}

- Volonté de développer une relation significative

- Importance d'être perçus comme un modèle positif de figure masculine

- Souci de proposer des activités d'apprentissage signifiantes

- Recherche d'approbation auprès des garçons

Au regard du tableau 3, cette communication genrée prévaut principalement dans les discussions que certains enseignants entreprennent auprès des garçons : « Je sais bien 
que plusieurs écoutent ou font du hockey, donc je mets ça de l'avant quand ils arrivent en classe » (PE18-13-460).

Plusieurs enseignants disent accueillir les garçons en abordant avec eux des thèmes qui touchent les loisirs ou les sports, et ce, en les questionnant entre autres sur leurs intérêts pour les sports présentés à la télévision ou encore sur la pratique d'activités sportives dans le quotidien. En contexte d'apprentissage, comme l'expriment certains enseignants, leurs échanges avec les garçons sont empreints de dynamisme : ces enseignants modulent leur voix, se déplacent davantage en classe et se mettent à proximité du bureau des garçons lorsqu'ils interagissent avec eux. Qui plus est, afin de saluer les garçons à leur arrivée en classe ou encore lors de rencontres dans le corridor, des enseignants mentionnent avoir tendance à leur donner une poignée de main bien sentie. Ainsi, la communication genrée naît de la volonté nommée par des enseignants de développer une relation significative avec les garçons : le discours ambiant rappelle les difficultés scolaires vécues par ces derniers, ce que plusieurs enseignants perçoivent comme un rappel du rôle déterminant qu'ils ont à jouer auprès des garçons, et ce, tant d'un point de vue scolaire que social. D'ailleurs, les enseignants soutiennent l'importance pour eux d'être perçus comme un modèle positif de figure masculine auprès des garçons. Au dire de ces enseignants, cette communication genrée s'actualise à travers leur souci de proposer des activités d'apprentissage signifiantes pour les garçons, puisqu'elles sont développées dans la volonté de rejoindre leurs intérêts ou leurs préoccupations. Par ailleurs, des enseignants révèlent être en quête d'approbation, chercher à plaire aux garçons en ayant une attitude qui tient compte d'une certaine normalisation de ce qu'est un garçon adolescent. D'autres garçons ne correspondant pas au modèle en sont exclus.

L'interventionnisme genré. L'interventionnisme genré se décrit comme l'orientation des interventions par les enseignants auprès des garçons en fonction d'une approche centrée sur des stéréotypes masculins.

\section{Tableau 4}

Interventionnisme genré

Catégorie : Interventionnisme genré

Définition : Volonté d'orienter les interventions auprès des garçons en fonction de stéréotypes masculins 


\section{Propriétés :}

- Peu de tolérance à l'égard des comportements perturbateurs manifestés par les garçons comparativement aux comportements manifestés par les filles

- Projets d'apprentissage développés autour d'intérêts typiquement masculins

- Proposition d'activités pratiques ou de manipulation en classe

\section{Conditions d'existence :}

- Volonté d'offrir les meilleures interventions possibles

- Perceptions de différences d'attitudes entre les garçons et les filles

- Discours social ambiant à l'égard de la situation scolaire difficile des garçons

À la lumière du tableau 4, des enseignants témoignent être peu tolérants pour les comportements perturbateurs manifestés par les garçons : «Je dois dire que je ne suis pas très patient ou très tolérant avec eux parce que je sais bien que ça peut dégénérer vite. Je ne leur donne pas long de corde, comme on dit » (PE14-21-352).

Selon des enseignants, les garçons ont des comportements plus extériorisés, ce qui a pour effet d'attirer davantage leur attention et de les obliger à intervenir plus auprès d'eux. Par ailleurs, certains enseignants font part de leurs interventions élaborées sur le plan des apprentissages en tenant compte d'intérêts typiquement masculins : «On ne se gêne pas pour faire écrire sur leurs intérêts... les autos, le sport » (PE7-16-161). En effet, plusieurs enseignants rapportent que l'élaboration de projets d'apprentissage autour d'intérêts considérés comme typiquement masculins, comme les autos et le sport, est bénéfique pour les garçons, mais également pour l'ensemble de la classe puisqu'il s'agirait d'intérêts généralement peu exploités en classe.

Qui plus est, des enseignants mentionnent qu'ils proposent des activités pratiques ou de manipulation dans lesquelles les garçons sont invités à être actifs au cours de leurs apprentissages : « En leur demandant de trouver le volume d'un solide avec du sable, bien c'est plus concret pour eux » (PE6-9-139). D'autres enseignants disent ainsi que les garçons apprennent dans l'action, ce qui les encourage à offrir des interventions qui s'inscrivent dans la mise en pratique des éléments d'apprentissage théorique.

Cette volonté d'orienter les interventions en fonction du sexe de l'élève n'est pas sans fondement. Ainsi, selon les propos des enseignants, le fait d'intervenir différemment auprès des garçons par rapport aux filles témoigne d'une volonté d'offrir les meilleures interventions possibles et d'une perception que les garçons et les filles apprennent d'une manière qui est propre à leur sexe. Qui plus est, certains enseignants nous signalent que le discours ambiant à l'égard de la situation scolaire difficile des garçons n'est pas en reste 
pour expliquer l'existence de cet interventionnisme genré. Comme le soulèvent plusieurs enseignants, ce discours prend la forme d'une pression pour eux, car ils se sentent obligés d'agir compte tenu des attentes sociales et des collègues féminines qui voient d'un bon œil leur présence auprès des garçons.

\section{Les stéréotypes de genre véhiculés par les garçons}

Les stéréotypes de genre véhiculés par les garçons dans leurs perceptions déclarées à l'égard des relations qu'ils entretiennent avec les enseignants s'opérationnalisent en une catégorie, soit la distanciation émotive.

La distanciation émotive. La distanciation émotive est une perception stéréotypée des garçons voulant que les enseignants vivent moins d'émotions que les enseignantes.

\section{Tableau 5}

\section{Distanciation émotive}

Catégorie : Distanciation émotive

Définition : Perception stéréotypée des garçons par rapport au fait que les enseignants vivraient moins d'émotions que les enseignantes

Propriétés :

- Faible démonstration émotive (pleurs, cris, colère, etc.) des enseignants

- Peu d'importance accordée par les enseignants aux manifestations émotives des garçons

- Relativisme absolu pour ce qui est des situations émotives vécues à la fois du point de vue des garçons et de celui des enseignants eux-mêmes

- Dédramatisation de la part des enseignants concernant les situations jugées difficiles par les garçons en classe

\section{Conditions d'existence :}

- Éducation reçue par les enseignants

- Croyance des garçons selon laquelle les hommes ne sont pas prédisposés comme les femmes aux états émotifs

- Comparaison non nuancée de la part des garçons en ce qui a trait à l'expression émotive des enseignants et des enseignantes

- Perceptions négatives des garçons à l'égard de la manifestation d'émotions

Le tableau 5 démontre que les enseignants manifestent peu leurs émotions devant les garçons. L'exemple qui suit renforce ce constat : « Mes enseignants n'ont pas trop de 
réactions en classe. J'en ai jamais vu brailler ou crier... ou être ben fâché. C'est tout le contraire chez les enseignantes » (G40-8-2078).

Au dire de certains garçons, les enseignants accordent bien peu d'importance aux manifestations émotives des garçons en classe, relativisent de manière absolue toute situation émotive vécue, à la fois par les garçons que par eux-mêmes, et dédramatisent les situations jugées difficiles en classe : «Les enseignants, c’est jamais grave. Tu brailles ta vie parce que tu feeles pas, mais là ils disent que c'est pas grave, que tu vas feeler mieux et que t'as pas besoin de brailler pour ça, de prendre sur moi... » (G14-22-1979).

Ce stéréotype voulant que les enseignants soient libres de l'émotivité provient, comme le verbalisent certains garçons, de l'éducation que les enseignants ont reçue : «Sont de même parce que c'était de même quand ils étaient à notre âge. Les gars, c'est moins.... démon... démonstra... [un autre élève complète : "démonstratifs"] » (G81-12-3097).

En outre, plusieurs garçons soulèvent entretenir la croyance voulant que les hommes ne soient pas prédisposés comme les femmes aux états émotifs : «On vient peutêtre au monde de même. Les enseignants ont pas tant d'émotions que les enseignantes... c'est peut-être dans les gènes »(G17-22-1452). D'ailleurs, certains garçons parviennent difficilement à comparer de manière nuancée l'expression émotive des enseignants à celle des enseignantes. Cette position très polarisée renforce le stéréotype selon lequel les enseignants contrôlent leurs émotions comparativement aux enseignantes : « Bah ! je dirais que les enseignants sont de même, là ; pas très trop contents, pas trop fâchés, c'est ben égal. On dirait que de l'autre bord les enseignantes sont pognées avec des hauts et des bas » (G51-12-2390).

En dernier lieu, certains garçons perçoivent négativement les manifestations d'émotions, que ce soit de la part des enseignants ou d'eux-mêmes. Dans ce contexte, ils sont plus enclins à reconnaître certains de leurs enseignants comme étant en contrôle de leurs émotions compte tenu de leur volonté d'être comme eux : « J'ai pas l'impression que c'est bien bon de se faire mener par ses émotions. J'ai pas l'impression d'être ben ben quelqu'un d'émotif... Comme les autres gars. C'est une affaire de filles, ça. C'est pareil pour les enseignants, là... » (G28-7-1760). 


\section{Discussion}

Les résultats présentés dans cet article mettent en évidence que les stéréotypes de genre prévalent dans les perceptions déclarées de certains participants à l'égard des relations qu'ils entretiennent mutuellement.

D'une part, les propos de certains garçons nous montrent que ces derniers entretiennent une perception stéréotypée selon laquelle les enseignants vivraient moins d'émotions que les enseignantes. Ce stéréotype de genre est mis en évidence par des garçons qui rapportent que leurs enseignants démontrent peu leurs émotions en leur présence. Ce constat concorde avec le phénomène d'atrophie émotive qui peut être perçu chez les hommes (Deslauriers, Baron et al., 2019). En d'autres mots, cela rejoindrait le stéréotype de genre qui reconnaît que certains types d'hommes ne vivent pas leurs émotions ou les contrôlent ; par exemple, qu'ils ne pleurent pas publiquement et qu'ils envisagent les événements d'une manière rationnelle (Blais et al., 2019). D'ailleurs, plusieurs auteurs s'entendent pour dire que la prégnance de ce stéréotype chez les hommes demeure néfaste puisqu'il les isole lorsqu'ils éprouvent des difficultés personnelles : ils n'auront pas le réflexe d'aller chercher de l'aide ou du soutien, puisqu'on attend socialement d'eux qu'ils traversent aisément les épreuves (Tremblay et al., 2015). En effet, certains hommes auraient toujours autant de difficulté à demander de l'aide, ce qui s'avère certainement un élément sur lequel il serait pertinent de se pencher, considérant que les conclusions de notre étude ne permettent pas de nous avancer sur les conséquences possibles de l'atrophie émotive des enseignants dans leurs relations avec les garçons. Il est toutefois intéressant de noter que d'autres chercheurs ont remarqué que les hommes relativement plus jeunes sont plus à l'aise de parler de leurs émotions que les plus âgés (Tremblay et al., 2016). Cela dit, il aurait sans doute été profitable de mettre en perspective les propos tenus par certains garçons concernant leurs enseignants selon l'âge de ces derniers. De fait, cet exercice nous aurait peut-être permis d'associer les dires des garçons à une tranche d'âge d'enseignants en particulier, et donc de nous situer plus justement avec les résultats de Tremblay et al. (2016).

D'autre part, la distanciation émotive des enseignants, rapportée par les garçons pour décrire entre autres le peu d'importance qu'accordent les enseignants à leurs manifestations émotives, trouve écho dans les travaux d'Anderson (2009) et de Connell (1995) mentionnés précédemment. Néanmoins, bien que nos résultats mettent en 
évidence la question de la distanciation émotionnelle des hommes à l'égard des autres hommes, soit des élèves de sexe masculin dans le cas qui nous occupe, ils ne montrent pas l'évidence d'une forme de distanciation physique s'opérant entre les enseignants et les garçons. Au contraire, lorsqu'on s'intéresse aux résultats relatifs à la communication genrée, on remarque que les enseignants évoquent manifester des signes de proximité physique dans leurs relations avec les garçons, notamment en échangeant des poignées de main ou en étant à proximité des bureaux des garçons quand ils interagissent avec eux. Cette proximité physique, contraire à l'archétype masculin traditionnel décrit par Anderson (2009) et Connell (1995), pourrait s'expliquer par le fait que les comportements entre les hommes se révéleraient plus affectueux et moins distants depuis la dernière décennie (McCormack, 2011).

Qui plus est, comme le rapportent des enseignants, ils ont la volonté de développer une relation significative avec les garçons. Si cette volonté est ainsi exprimée, cela laisse croire que le personnel enseignant considère les élèves sur les plans individuel et scolaire (Tardif et Gauthier, 2017) et que la REÉ entre les enseignants et les garçons se détacherait de la vision de Durkheim (2014), vision qui conçoit la REÉ dans un rapport de pouvoir du personnel enseignant sur les élèves. Toutefois, il faut certainement user de prudence vis-à-vis d'un tel constat parce que, comme le soulignent Postic (2001) et Rayou (2017), l'école demeure un agent de reproduction sociale susceptible de perpétuer un système d'attitudes ou de manières de penser, ce qui semble d'ailleurs être le cas puisque nos résultats montrent que certains stéréotypes de genre persistent de nos jours en classe. Ainsi, cette tentative pour développer une relation significative telle qu'énoncée par les enseignants auprès des garçons pourrait se décrire comme étant maladroite puisque, bien qu'elle véhicule des stéréotypes masculins négatifs (p. ex., atrophie émotive), elle ne permettrait pas, de toute manière, de rejoindre l'ensemble des garçons puisque ces derniers ne partagent pas tous les mêmes intérêts, comme le sport ou les autos. En développant une REÉ sur la base de stéréotypes de genre masculins, il n'en demeure pas moins que l'on engendrerait des laissés-pour-compte, c'est-à-dire des garçons qui n'incarnent pas ou ne se reconnaissent pas dans les stéréotypes de genre masculins véhiculés par leurs enseignants.

Par ailleurs, nous sommes d'avis que les stéréotypes de genre (p. ex., l'interventionnisme et la communication genrés) entretenus par les enseignants à l'endroit des garçons seraient le fruit du discours social ambiant qui martèle que la présence de 
plus d'hommes en enseignement agirait positivement sur la réussite scolaire des garçons (Royer, 2010, 2011). Même s'il faut rappeler qu'à notre connaissance la documentation scientifique ne nous renseigne pas sur la présence d'un lien significatif entre le sexe de l'enseignant et la réussite scolaire des garçons, cette croyance en une réussite scolaire passant par une présence plus marquée d'hommes en enseignement nous apparaît réductrice, considérant la pluralité des dimensions qui peuvent entrer en jeu dans le développement et le maintien de la réussite scolaire (p. ex., dispositions personnelles, qualité de la relation enseignant-élève, soutien adéquat des parents). De plus, elle pourrait être interprétée comme une remise en question du travail qu'exercent déjà les enseignantes auprès des garçons, les incitant à s'engager, à persévérer et à réussir à l'école.

\section{Conclusion}

Cet article avait pour but d'identifier les stéréotypes de genre véhiculés par les enseignants et les garçons à l'égard de la REÉ au secondaire. À la lecture des propos des enseignants et des garçons, il nous a été donné de voir que des stéréotypes de genre persistent en classe, notamment sur le plan de la communication et de l'intervention en classe.

Sur le plan des limites de cette recherche, mentionnons que nous avons demandé aux participants d'appuyer leurs dires sur leurs expériences vécues durant l'année scolaire en cours. À ce sujet, nous ne pouvons garantir que les garçons se soient limités à décrire leurs relations sur cette période : il est probable que certains d'entre eux aient puisé dans leurs souvenirs du primaire ou de la première année du secondaire afin d'étayer leurs perceptions actuelles. Cette limite est également valable pour les enseignants, qui ont pu faire part d'éléments anecdotiques ne relevant pas de la dynamique actuelle de leurs classes. Qui plus est, il est important de reconnaître que les analyses effectuées dans la présente étude n'ont pas fait de distinction entre les types de classes d'adaptation scolaire ou sociale et d'enseignement régulier, ce qui s'avérerait une perspective de recherche pertinente pour examiner si les mêmes stéréotypes de genre à l'égard de la REÉ y sont véhiculés. 
Enfin, il serait sans doute judicieux que le personnel enseignant et les élèves s'engagent dans une réflexion sur l'incidence des stéréotypes de genre dans leurs relations. D'ailleurs, il est à penser que le personnel enseignant aurait tout intérêt à se défaire de ses perceptions genrées concernant les élèves et à s'assurer plutôt de concevoir leur unicité et leur hétérogénéité sur les plans affectif, social et cognitif, et ce, sans égard à leur sexe. Dès lors, peut-être arriverons-nous à l'établissement d'une relation enseignant-élève de qualité et à une réussite scolaire plus marquée pour l'ensemble des élèves, et plus particulièrement pour les garçons. 


\section{Références}

Anderson, E. (2009). Inclusive masculinity: The changing nature of masculinities. Routledge.

Auwärter, A. E. et Aruguete, M. S. (2008). Effects of student gender and socioeconomic status on teacher perceptions. The Journal of Educational Research, 101(4), 242-246. https://doi.org/10.3200/JOER.101.4.243-246

Baby, A. (2016). L'école québécoise : un nouvel humanisme pour tous. Dans P. Doray et C. Lessard (dir.), 50 ans d'éducation au Québec (p. 229-234). Presses de l’Université du Québec.

Bianco, M. et Leech, N. L. (2010). Twice-exceptional learners: Effects of teacher preparation and disability labels on gifted referrals. Teacher Education and Special Education, 33(4), 319-334. https://doi.org/10.1177/0888406409356392

Blais, A. et Durand, C. (2009). Le sondage. Dans B. Gauthier (dir.), Recherche sociale : de la problématique à la collecte des données (5e éd., p. 445-478). Presses de l’Université du Québec.

Blais, M., Lavigne, J. et Médico, D. (2019). La masculinité à l'épreuve de la diversité des sexualités, des corps et des genres. Dans J.-M. Deslauriers, M. Lafrance et G. Tremblay (dir.), Réalités masculines oubliées (p. 43-65). Presses de l'Université Laval.

Bowlby, J. (1969). Attachment and Loss, Vol. 1: Attachment. Basic Books.

Bréau, A. et Lentillon-Kaestner, V. (2016). Lutter contre les stéréotypes de genre : un enjeu d'actualité et d'égalité pour l'École. L'Éducateur, 5, 32-34. http://hdl. handle.net/20.500.12162/1151

Burusic, J., Babarovic, T. et Seric, M. (2012). Differences in elementary school achievement between girls and boys: Does the teacher gender play a role? European Journal of Psychology of Education, 27(4), 523-538. https://doi. org/10.1007/s 10212-011-0093-2

Carrington, B., Francis, B., Hutchings, M., Skelton, C., Read, B. et Hall, I. (2007). Does the gender of the teacher really matter? Seven- to eight-year-olds' accounts of 
their interactions with their teachers. Educational Studies, 33(4), 397-413. https:// doi.org/10.1080/03055690701423580

Carrington, B., Tymms, P. et Merrell, C. (2008). Role models, school improvement and the 'gender gap' - do men bring out the best in boys and women the best in girls? British Educational Research Journal, 34(3), 315-327. https://doi. org/10.1080/01411920701532202

Castillo-Mayén, R. et Montes-Berges, B. (2014). Análisis de los estereotipos de género actuales. Anales de psicología, 30(2), 1044-1060. https://www.redalyc.org/ articulo.oa? $\mathrm{id}=16731690027$

Charmaz, K. (2006). Constructing grounded theory : A practical guide through qualitative analysis. SAGE.

Clark, M. A., Thompson, P. et Vialle, W. (2008). Examining the gender gap in educational outcomes in public education: involving pre-service school counsellors and teachers in cross-cultural and interdisciplinary research. International Journal for the Advancement of Counselling, 30(1), 52-66. http://dx.doi.org/10.1007/ s10447-007-9044-7

Clément, P., Dreux, G., Laval, C. et Vergne, F. (2011). La nouvelle école capitaliste. La Découverte.

Connell, R. W. (1995). Masculinities. University of California Press.

Davis, E. A. et Miyake, N. (2004). Explorations of scaffolding in complex classroom systems. The Journal of the Learning Sciences, 13(3), 265-272. https://doi. org/10.1207/s15327809jls1303_1

Davis, G. A. et Rimm, S. B. (2004). Education of the gifted and talented (5e éd.). Allyn \& Bacon.

Deslauriers, J.-M., Baron, M. et Negura, L. (2019). Les hommes souffrant de dépression : un problème méconnu. Dans J.-M. Deslauriers, M. Lafrance et G. Tremblay (dir.), Réalités masculines oubliées (p. 183-214). Presses de l’Université Laval.

Durkheim, É. (2014). L'évolution pédagogique en France (3e éd.). Presses universitaires de France. 
Duru-Bellat, M. (2016). À l'école du genre. Enfances \& Psy, 69(1), 90-100. https://doi. org/10.3917/ep.069.0090

Erbas, D., Turan, Y., Aslan, Y. G. et Dunlap, G. (2010). Attributions for problem behavior as described by Turkish teachers of special education. Remedial and Special Education, 31(2), 116-125. https://doi.org/10.1177/0741932508327461

Forquin, J.-C. (2008). Sociologie du curriculum. Presses universitaires de Rennes.

Francis, B. (2008). Teaching manfully? Exploring gendered subjectivities and power via analysis of men teachers' gender performance. Gender and Education, 20(2), 109-112. https://doi.org/10.1080/09540250701797226

Geoffrion, P. (2009). Le groupe de discussion. Dans B. Gauthier (dir.), Recherche sociale : de la problématique à la collecte de données (p. 391-414). Presses de l’Université du Québec.

Glaser, B. G. et Strauss, A. L. (1967). The discovery of grounded theory: Strategies for qualitative research. Aldine de Gruyter.

Good, T. et Brophy, J. (2017). Looking in classroom (11e éd.). Allyn and Bacon.

Hansen, M. et Quintero, D. (2018, 10 juillet). How gender diversity among the teacher workforce affects student learning. Brookings. https:// www.brookings.edu/blog/brown-center-chalkboard/2018/07/10/ how-gender-diversity-among-the-teacher-workforce-affects-student-learning/

Harrison, N. (2011). Teaching and learning in Aboriginal education (2e éd.). Oxford University Press.

Homsy, M. et Savard, S. (2018, avril). Décrochage scolaire au Québec : dix ans de surplace, malgré les efforts de financement [note de recherche]. Institut du Québec. https://www.rumeurduloup.com/wp-content/uploads/2020/02/9652 d\%C 3\%A9crochage-scolaire-au-qu\%C $3 \%$ A9bec idq_br.pdf

Karsenti, T. et Savoie-Zajc, L. (dir.). (2018). La recherche en éducation : étapes et approches (4e éd. revue et mise à jour). Presses de l'Université de Montréal.

Lamarre, S. (2019). Les hommes au sein de l'école primaire québécoise. Dans J.-M. Deslauriers, M. Lafrance et G. Tremblay (dir.), Réalités masculines oubliées (p. 125-149). Presses de l’Université Laval. 
Legewie, J. et DiPrete, T. A. (2012). School context and the gender gap in educational achievement. American Sociological Review, 77(3), 463-485. https://doi. org/10.1177/0003122412440802

Lips, H. M. (2005). Masculinity and femininity: Myths and stereotypes. Dans H. M. Lips (dir.), Sex \& gender: An introduction (5e éd., p. 1-53). McGraw-Hill.

McBee, M. T. (2006). A descriptive analysis of referral sources for gifted identification screening by race and socioeconomic status. Journal of Secondary Gifted Education, 17(2), 103-111. https://doi.org/10.4219/jsge-2006-686

McCormack, M. (2011). Hierarchy without hegemony: Locating boys in an inclusive school setting. Sociological Perspectives, 54(1), 83-101. https://doi.org/10.1525/ sop.2011.54.1.83

McGrath, K. et Sinclair, M. (2013). More male primary-school teachers? Social benefits for boys and girls. Gender and Education, 25(5), 531-547. https://doi.org/10.1080 $\underline{109540253.2013 .796342}$

Ministère de l'Éducation et de l'Enseignement supérieur [MEES]. (2018). Personnel des commissions scolaires, selon le statut et la catégorie d'emploi, le secteur et l'ordre d'enseignement et le sexe, Québec, de 2010-2011 à 2015-2016. Gouvernement du Québec.

Montandon, C. (2002) Approches systémiques des dispositifs pédagogiques : enjeux et méthodes. L'Harmattan.

Mosconi, N. (2017). Genre et éducation des filles. Des clartés de tout. L'Harmattan.

Numa-Bocage, L. (2007). La médiation didactique : un concept pour penser les registres d'aide de l'enseignant. Carrefours de l'éducation, 23(1), 55-70. https://doi. org/10.3917/cdle.023.0055

Paillé, P. et Mucchielli, A. (2008). L'analyse qualitative en sciences humaines et sociales (2e éd.). Armand Colin.

Paillé, P. et Mucchielli, A. (2016). L'analyse qualitative en sciences humaines et sociales (4e éd.). Armand Colin.

Passerieux, V. (2010). L'intello ou le bouffon. Diversité, (162), 91-95. 
Pianta, R. C., Hamre, B. K. et Mintz, S. (2012). Classroom Assessment Scoring SystemSecondary (CLASS-S). University of Virginia.

Postic, M. (2001). La relation éducative. Presses universitaires de France.

Prentice, D. A. et Carranza, E. (2002). What women and men should be, shouldn't be, are allowed to be, and don't have to be: The contents of prescriptive gender stereotypes. Psychology of women quarterly, 26(4), 269-281. https://doi. org/10.1111/1471-6402.t01-1-00066

Pujade-Renaud, C. (2005). Le corps de l'élève dans la classe. L'Harmattan.

Quenzel, G. et Hurrelmann, K. (2013). The growing gender gap in education. International Journal of Adolescence and Youth, 18(2), 69-84. https://doi.org/10.1 $\underline{080 / 02673843.2012 .665168}$

Qvortrup, J. (1995). From useful to useful: The historical continuity of children's constructive participation. Sociological Studies of Children, 7(1), 49-76.

Rayou, P. (1998). La Cité des lycéens. L’Harmattan.

Rayou, P. (2017). Sociologie de l'éducation. Presses universitaires de France.

Rey, B. (1999). Faire la classe à l'école élémentaire. ESF.

Riley, T. et Ungerleider, C. (2012). Self-fulfilling prophecy: How teachers' attributions, expectations, and stereotypes influence the learning opportunities afforded Aboriginal students. Canadian Journal of Education, 35(2), 303-333. https:// journals.sfu.ca/cje/index.php/cje-rce/article/view/406

Royer, É. (2010). Leçons d'éléphants : pour la réussite des garçons à l'école. École et comportement.

Royer, É. (2011, 23-25 mars). Ô Roméo ! Ô Roméo ! Pourquoi es-tu Roméo ? De la difficulté des garçons avec l'école. Communication présentée au 36e congrès annuel de l'Association québécoise des troubles d'apprentissage (AQETA), Montréal.

Savoie-Zajc, L. (2010). L'entrevue semi-dirigée. Dans B. Gauthier (dir.), Recherche en sciences sociales : de la problématique à la collecte des données (5e éd., $\mathrm{p}$. 337-360). Presses de l’Université du Québec. 
Savoie-Zajc, L. (2018). La recherche qualitative/interprétative. Dans T. Karsenti et L. Savoie-Zajc (dir.), La recherche en éducation : étapes et approches (4e éd., p. 191-217). Presses de l’Université de Montréal.

Skibbe, L., Behnke, M. et Justice, L. M. (2004). Parental scaffolding of children's phonological awareness skills : Interactions between mothers and their preschoolers with language difficulties. Communication Disorders Quarterly, 25(4), 189-203. https://doi.org/10.1177/15257401040250040401

Strauss, A. L. et Corbin, J. (2004). Les fondements de la recherche qualitative : techniques et procédures de développement de la théorie enracinée (M.-H. Soulet et S. Emery, trad.). Academic Press ; Saint-Paul.

Tardif, M. et Gauthier, C. (2017). La pédagogie de demain. Dans C. Gauthier et M. Tardif (dir.), La pédagogie : théories et pratiques de l'Antiquité à nos jours (4e éd., p. 264-272). Gaëtan Morin.

Tremblay, G., Roy, J., Beaudet, L., Chamberland, L., Dupéré, S., Le Gall, J., Roy, V., Guilmette, D., Sirois-Marcil, J., Bizot, D., Lajeunesse, S.-L. et Desjardins, J. (2016). Les hommes et les services sociaux de la santé. Analyse qualitative d'entrevues de groupes focalisées tenues auprès d'hommes québécois. Masculinités et Société. http://www.perceptions.svs.ulaval.ca/sites/perceptions. svs.ulaval.ca/files/rapportgroupesdediscussionavecisbn_1fevrier2016.pdf

Tremblay, G., Roy, J., de Montigny, F., Séguin, M., Villeneuve, P., Roy, B., Guilmette, D., Sirois-Marcil, J. et Émond, D. (2015, juillet). Où en sont les hommes québécois en 2014 ? Sondage sur les rôles sociaux, les valeurs et sur le rapport des hommes québécois aux services [Rapport de recherche]. Masculinités et Société. https:// cerif.uqo.ca/sites/cerif.uqo.ca/files/sondageprojet_perceptions_version_finale.pdf

Van Ewijk, R. (2011). Same work, lower grade? Student ethnicity and teachers' subjective assessments. Economics of Education Review, 30(5), 1045-1058. https://doi.org/10.1016/j.econedurev.2011.05.008

Willis, P. (2011). L'école des ouvriers. Comment les enfants d'ouvriers obtiennent des boulots d'ouvriers. Agone. 